\title{
Ultra-Wideband Robust RCS Reduction with Triangle-type AMC Structure
}

\author{
Tao SONG, Lili CONG, Chuangming TONG \\ Air and Missile Defense Institute, Air Force Engineering University, Xi'an 710077, China \\ 475559422@qq.com,1183068955@qq.com,cmtong@126.com \\ Submitted November 3, 2017 / Accepted April 25, 2018
}

\begin{abstract}
A novel planar artificial magnetic conductor (AMC) structure is presented for ultra-wideband radar cross section reduction (RCSR). The structure consists of three different AMC tiles in a quadruple triangle-type chessboard configuration. By precise design, a $180^{\circ} \pm 30^{\circ}$ reflection phase difference between every two of the three units nearly covers from $3.98 \mathrm{GHz}$ to $18.84 \mathrm{GHz}$ to broaden the RCSR bandwidth. By this means, the scattered energy is redirected into several lobes and the measured RCSR for over $-6 \mathrm{~dB}$ is achieved nearly from $3.62 \mathrm{GHz}$ to $18 \mathrm{GHz}$ with a relative bandwidth of $133.02 \%$ for both polarizations. Both full-wave simulation and measurement results verify the capability and potentiality of the proposed design for ultra-wideband RCSR.
\end{abstract}

\section{Keywords}

Ultra-wideband, artificial magnetic conductor, polarization independent, radar cross section reduction, quadruple triangle chessboard

\section{Introduction}

With the rapid development of stealth and detection technology, there is an urgent need for electromagnetic invisibility or transparency materials, especially in military applications [1-4]. Artificial magnetic conductor (AMC), as a typical artificial composite structure, has been extensively applied in antenna radar cross section reduction (RCSR) [5-9] on its advantages of low profile, easy processing and good conformal shape. By combing AMC and perfect electric conductor (PEC) in a chessboard configuration, a $180^{\circ}$ phase difference between reflected waves from these two different tiles can be created [10], [11]. Consequently, the destructive interference between the reflected waves then results in RCSR in specular direction. However, the narrow band behavior of AMC structure resulting from its inherent resonance characteristic limits its wide application. In general, there are mainly two methods proposed in the previous literatures to achieve broadband RCSR. One is to design broadband AMC structures by multilayers [12], low relative permittivity and thick substrate [13], [14] and integration with active elements [15]. The other is to replace the conventional PEC tiles with another different AMC tiles to broaden the effective $180^{\circ}$ phase differential bandwidth [16-22]. Two different-shaped AMC structures were introduced in a checkerboard surface to obtain a $-10 \mathrm{~dB}$ RCSR bandwidth of $41 \%$ and $85 \%$, respectively in [19], [20]. However, the scattered fields were redirected into four strong lobes to the disadvantage of bistatic RCSR. In [21], two different types of non-absorptive AMC structures were arranged in an aperiodic fashion to distribute the scattered energy into six lobes, and a $-6 \mathrm{~dB}$ RCSR bandwidth of $66.67 \%$ is achieved as a result. Similarly, in [22], two different-sized Jerusalem Cross structures were presented in a triangular checkerboard configuration to redirect the power into eight main lobes with a -10dB RCSR bandwidth of $43 \%$. Nonetheless, the RCSR bandwidth is relatively narrow and the proposed structures cannot work well at lower frequencies. Moreover, when it comes to antenna array RCSR, especially for closely-spaced array, the problem that limited space between array elements cannot accommodate so many AMC units may arise, let alone for ultra-wideband RCSR. How to increase space utilization, in other words, to optimize the layout of different AMCs to achieve ultra-wideband RCSR of antenna array becomes an urgent problem. Hence, this paper is presented to provide a feasible approach to address the problems mentioned above.

In this paper, a quadruple-triangle-type chessboard configuration consisting of three different AMC tiles is proposed to achieve destructive phase difference over an ultra-wideband. By precise design, a $180^{\circ} \pm 30^{\circ}$ reflection phase difference between every two of the three units nearly covers from $3.98 \mathrm{GHz}$ to $18.84 \mathrm{GHz}$ to broaden the RCSR bandwidth. In this way, over $-3 \mathrm{~dB}$ RCSR is achieved nearly from $4.4 \mathrm{GHz}$ to $22 \mathrm{GHz}$ for both polarizations, whilst the scattered energy is redirected into several lobes to the benefit of bistatic RCSR. The prototype is fabricated and measured. The measured results show that an almost $133.02 \%$ relative bandwidth for over $-6 \mathrm{~dB}$ RCSR is achieved nearly from $3.62 \mathrm{GHz}$ to $18 \mathrm{GHz}$ for both polarizations, which verifies the capability and potentiality of the proposed design for ultra-wideband RCSR. 


\section{Design Principles}

To clearly interpret the mechanism of RCSR, a simplest conventional-chessboard metamaterial (MTM) array consisting of $2 \times 2$ elements is depicted in Fig. 1(a) and the total scattering field can be described as [23]:

$$
\boldsymbol{E}=\boldsymbol{E}_{\mathrm{MTM} 1} \cdot A F_{\mathrm{MTM} 1}+\boldsymbol{E}_{\mathrm{MTM} 2} \cdot A F_{\mathrm{MTM} 2}
$$

where $\boldsymbol{E}$ and $A F$ represent the scattered pattern and array factor respectively.

$$
\begin{gathered}
\boldsymbol{E}_{\mathrm{MTM} 1}=E_{1} \exp \left(\mathrm{j} \varphi_{1}\right) \boldsymbol{E}_{\text {element } 1}, \\
\boldsymbol{E}_{\mathrm{MTM} 2}=E_{2} \exp \left(\mathrm{j} \varphi_{2}\right) \boldsymbol{E}_{\text {element2 }}, \\
A F_{\mathrm{MTM} 1}=\exp [\mathrm{j}(k x+k y) d / 2]+\exp [\mathrm{j}(-k x-k y) d / 2], \\
A F_{\mathrm{MTM} 2}=\exp [\mathrm{j}(-k x+k y) d / 2]+\exp [\mathrm{j}(k x-k y) d / 2]
\end{gathered}
$$

where $E_{1}$ and $E_{2}$ are amplitudes of the scattering field, $\boldsymbol{E}_{\text {element1 }}$ and $\boldsymbol{E}_{\text {element2 }}$ are polarized direction, $\varphi_{1}$ and $\varphi_{2}$ are phase shifts, $d$ is the distance between adjacent elements, $k x=2 \pi \sin \theta \cos \varphi / \lambda, k y=2 \pi \sin \theta \sin \varphi / \lambda$.

As for our proposed design in Fig. 1(d), the total scattering field can be described as

$$
\begin{aligned}
\boldsymbol{E}= & \boldsymbol{E}_{\mathrm{MTM} 1} \cdot A F_{\mathrm{MTM} 1}+\boldsymbol{E}_{\mathrm{MTM} 2} \cdot A F_{\mathrm{MTM} 2}+ \\
& \boldsymbol{E}_{\mathrm{MTM} 3} \cdot A F_{\mathrm{MTM} 3}+\boldsymbol{E}_{\mathrm{MTM} 4} \cdot A F_{\mathrm{MTM} 4} .
\end{aligned}
$$

It is clearly that the distance from the central point of each AMC tile to the origin is the same, and then we assume it to be $d$. The total scattering field is then described as

$$
\begin{aligned}
\boldsymbol{E}= & E_{1} \mathrm{e}^{\mathrm{j} \varphi_{1}} \boldsymbol{E}_{\text {element1 }} \mathrm{e}^{\mathrm{j} S_{1}}+E_{2} \mathrm{e}^{\mathrm{j} \varphi_{2}} \boldsymbol{E}_{\text {element2 }} \mathrm{e}^{\mathrm{j} S_{2}}+ \\
& E_{3} \mathrm{e}^{\mathrm{j} \varphi_{3}} \boldsymbol{E}_{\text {element3 }} \mathrm{e}^{\mathrm{j} S_{3}}+E_{4} \mathrm{e}^{\mathrm{j} \varphi_{4}} \boldsymbol{E}_{\text {element4 }} \mathrm{e}^{\mathrm{j} S_{4}}
\end{aligned}
$$

where $S_{1}=-d \sin \theta \cos \varphi, S_{2}=d \sin \theta \sin \varphi, S_{3}=d \sin \theta \cos \varphi$, $S_{4}=-d \sin \theta \sin \varphi$.

For monostatic RCS with incident wave vertically impinging on the MTM array, the scattering field would be the same with the incident one, and then $\mathbf{E}_{\text {element } 1}=$ $\mathbf{E}_{\text {element2 }}=\mathbf{E}_{\text {element3 }}=\mathbf{E}_{\text {element4}}$. Assuming that each element totally reflects the incident wave with no absorption, then $E_{1}=E_{2}=E_{3}=E_{4}$. For normal incidence with $(\theta, \varphi)=(0,0)$, the total scattering field can be further simplified as

$$
\boldsymbol{E}=E_{1}\left(2 \mathrm{e}^{\mathrm{j} \varphi_{1}}+\mathrm{e}^{\mathrm{j} \varphi_{2}}+\mathrm{e}^{\mathrm{j} \varphi_{4}}\right) \boldsymbol{E}_{\text {element1 }} .
$$

To achieve 10-dB RCSR in normal direction in comparison with ideal scattering field with all unit cells in phase, there will be

$$
|\boldsymbol{E}|^{2} /\left|\boldsymbol{E}_{0}\right|^{2} \leq-10 \mathrm{~dB}
$$

then

$$
\left|2+\exp \left[\mathrm{j}\left(\varphi_{2}-\varphi_{1}\right)\right]+\exp \left[\mathrm{j}\left(\varphi_{4}-\varphi_{1}\right)\right]\right|^{2} \leq 1.6
$$

Assume that $\varphi_{2}-\varphi_{1}=\varphi_{4}-\varphi_{1}=\Phi$, then equation (10) can be simplified as
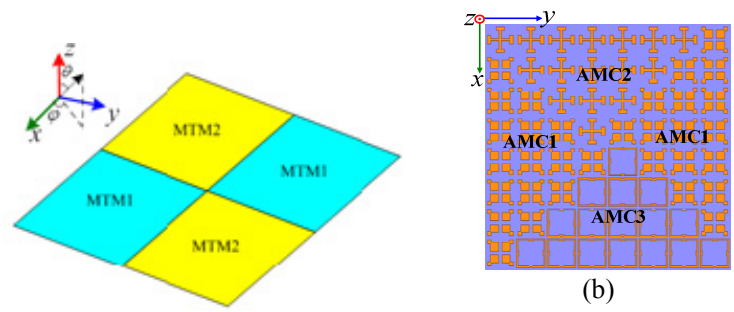

(b)
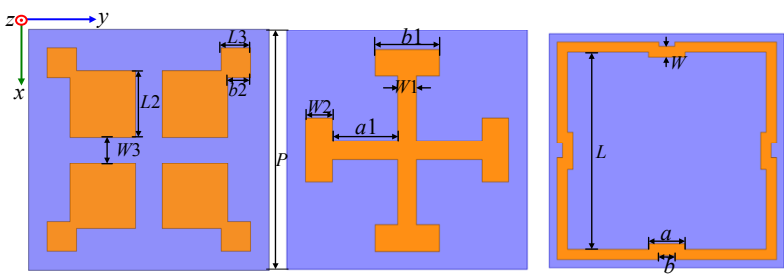

(c)

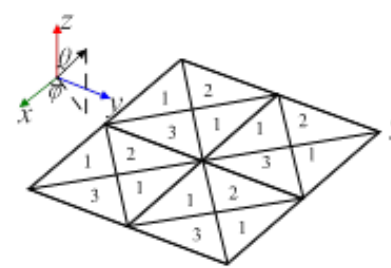

(d)

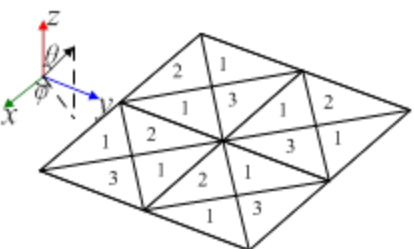

(e)
Fig. 1. (a) Schematic of a traditional $2 \times 2$ MTM array; (b) quadruple-triangle arrangement of AMC1, AMC2 and AMC3 tiles; (c) unit cells of AMC1, AMC2 and AMC3; (d) basic unit arrangement of case1; (e) basic unit arrangement of case 2 .

$$
|1+\exp (\mathrm{j} \Phi)|^{2} \leq 0.4
$$

thus

$$
143^{\circ} \leq \Phi \leq 217^{\circ} \text {. }
$$

For simplicity, we consider $180^{\circ} \pm 30^{\circ}$ as effective phase difference for the following analysis. And it can be concluded that once the phase difference between $\varphi_{2}$ and $\varphi_{1}$, $\varphi_{3}$ and $\varphi_{1}$ varies in the range from $150^{\circ}$ to $210^{\circ}$, the scattering field can be canceled in the backward direction according to the phase cancellation principle [10]. Hence, a considerable RCSR can be achieved. It is worth noting that the proposed arrangement of sub-units (Fig. 1(b)) is not strictly symmetric around the origin point, which might cause a slight difference between sub-units analysis and the final planar arrangement. Therefore, the sub-units analysis is just used as a guidance and ideal analysis to evaluate the RCSR bandwidth, and then to facilitate the design process and easy-understanding of the working principle.

\section{Simulation and Discussion}

\subsection{Design and Analysis of the Unite Cell}

The each single sub-unit of the three different AMC tiles is depicted in Fig. 1(c), nominated as AMC1, AMC2 and AMC3, respectively. All of the three units are composed of two metallic layers separated by an exactly samesized substrate with a dielectric constant of 2.65 and a loss tangent of 0.002 . Moreover, despite of the top metallic 
layers with different shapes, all of the three units are backed by a full metallic ground with the same periodicity. To create destructive interference in the largest band-width, full-wave numerical simulations have been carried out to optimize the parameters in the Ansoft HFSS v.14.0. Floquet

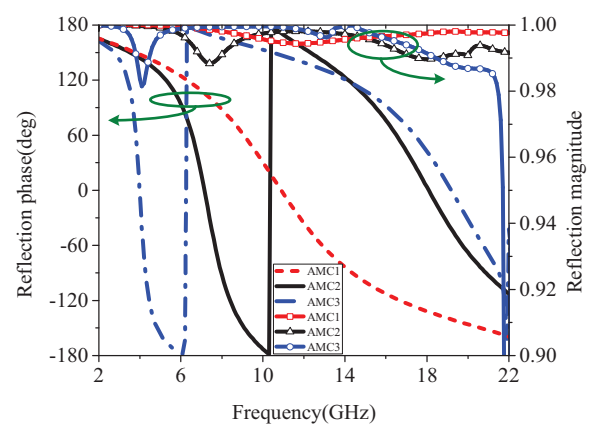

(a)

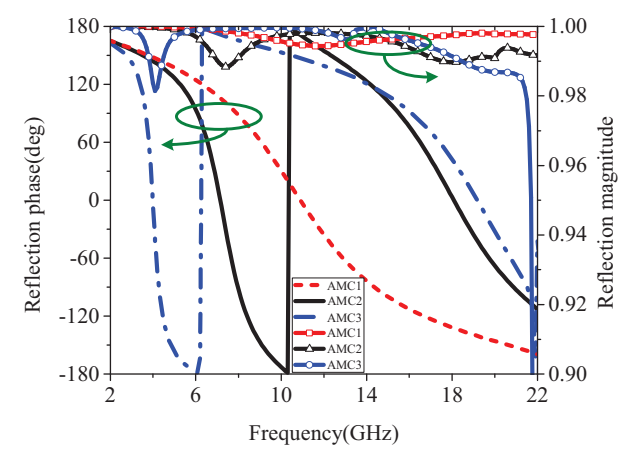

(b)

Fig. 2. Reflection characteristics of $\mathrm{AMC1}, \mathrm{AMC} 2$ and AMC3: (a) TE polarization; (b) TM polarization.

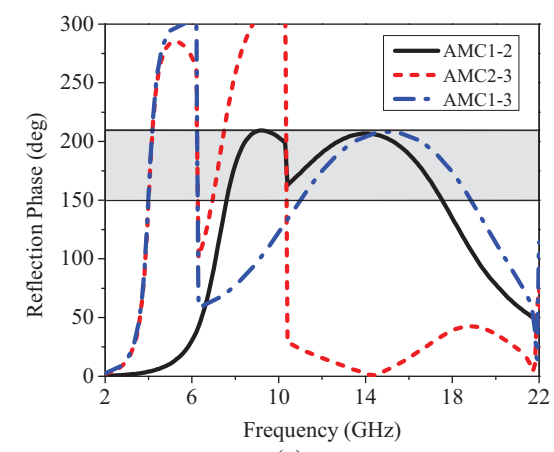

(a)

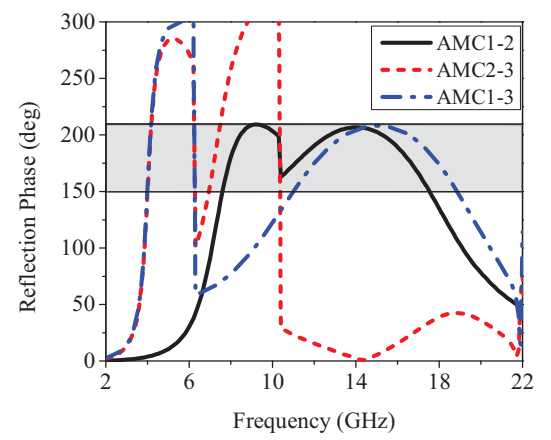

(b)

Fig. 3. Reflection phase differences between every two of the three AMC unit cells: (a) TE polarization; (b) TM polarization. port excitations and master/slave boundaries are performed on each single sub-unit to simulate the infinite $2 \mathrm{D}$ periodic boundary condition. The finally optimized parameters are as follows: $P=9 \mathrm{~mm}, a=1.4 \mathrm{~mm}, a_{1}=2.45 \mathrm{~mm}, b=$ $0.6 \mathrm{~mm}, b_{1}=2.4 \mathrm{~mm}, b_{2}=0.86 \mathrm{~mm}, W=0.4 \mathrm{~mm}, W_{1}=$ $0.7 \mathrm{~mm}, W_{2}=1 \mathrm{~mm}, W_{3}=1 \mathrm{~mm}, L_{1}=7.6 \mathrm{~mm}, L_{2}=$ $2.44 \mathrm{~mm}$ and $L_{3}=1.1 \mathrm{~mm}$.

Figure 2 and 3 plot the reflection characteristics of the three different AMC units against frequency as well as the phase differences between every two of them. As depicted in Fig. 2, when the normal incidence is TE polarized with electric field along y-axis, the reflection magnitudes maintain upon 0.98 from $2 \mathrm{GHz}$ to $21.5 \mathrm{GHz}$, indicating that the energy is almost reflected with no absorption. The simulated result meets the requirement to the elements in phase cancellation principle. Furthermore, it is clearly shown that AMC1 exhibits only one $0^{\circ}$ reflection phase point at $10.9 \mathrm{GHz}$, while AMC2 and AMC3 demonstrate dual $0^{\circ}$ reflection phase points at $7.15 \mathrm{GHz}$ and $18 \mathrm{GHz}, 3.98 \mathrm{GHz}$ and $19.18 \mathrm{GHz}$, respectively. Meanwhile, the phase difference covering the range of $180^{\circ} \pm 30^{\circ}$ between every two of the three AMC units nearly ranges from $3.98 \mathrm{GHz}$ to $18.84 \mathrm{GHz}$ except from $4.2 \mathrm{GHz}$ to $6.9 \mathrm{GHz}$. Therefore, we can infer that by combining the three different AMC units in an adequate configuration, the $180^{\circ}$ reflection phase difference bandwidth would be broadened, implying RCSR in a wider frequency band based on the phase cancellation principle. The situation of TM-polarized normal incidence with the electric field along $\mathrm{x}$-axis is the same with TE-polarized incidence, revealing obvious independence on the polarization of incident wave. The reason can be ascribed to the completely symmetric geometry of each single sub-unit.

\subsection{Ultra-Wide Band AMC Design}

For two different kind of AMC units, the classical chessboard arrangement has been extensively used to achieve broadband RCSR [16-20]. As for the three different AMC units proposed in this paper, a quadruple-triangle-type chessboard configuration is proposed to further broaden RCSR bandwidth and achieve bistatic RCSR simultaneously. In order to balance the RCSR performance in the low and high frequency band, on the basis of the simulated reflection phase differences shown in Fig. 3, the AMC1 is chosen as the mediator, which accounts for twoquarters of the quadruple-triangle configuration. In this way, the AMC1 tiles can be always surrounded by AMC2 and AMC3 tiles to guarantee good RCSR performance in as wide frequency band as possible.

In order to broaden RCSR bandwidth and achieve satisfactory RCSR performance simultaneously, two different arrangements named case 1 and case 2 have been considered. For case1, the basic unit is made up of the three AMC tiles in a quadruple-triangle configuration, as depicted in Fig. 1(b) and (d). As for case 2, by rotating the basic unit in case $190^{\circ}$ counter-clockwise around $z$-axis, we get the other basic unit. These two basic units are then orthogonally 


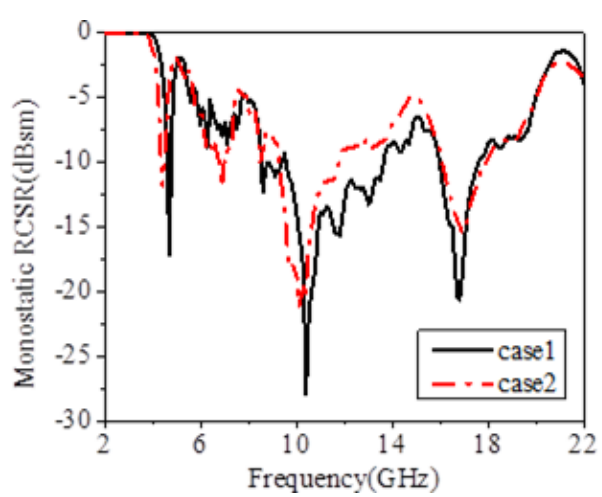

Fig. 4. Normalized RCSR comparison versus frequency under normally incident wave for case1 and case2.

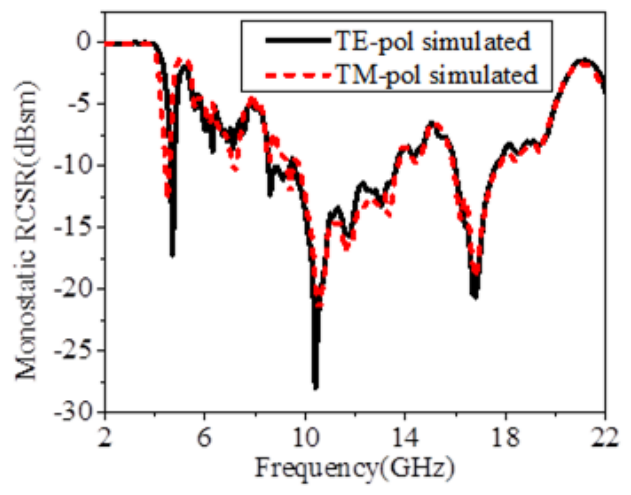

Fig. 5. RCSR versus frequency of the proposed design normalized by a same-sized metallic plate.

arranged with respect to its neighbor in a chessboard configuration as shown in Fig. 1(e). It is worth noting that the three AMC tiles can be arranged in other alternative configurations.

To evaluate the RCSR performance of the above two cases, the monostatic RCS against frequency under normal incident wave have been investigated for comparison. The simulated monostatic RCS value normalized by that of a completely metallic plate with exactly the same dimension is demonstrated in Fig. 4. For case 1, an ultra-wideband RCSR over $-6 \mathrm{~dB}$ is achieved from $4.52 \mathrm{GHz}$ to $4.86 \mathrm{GHz}$ (7.2\% bandwidth) and from $5.9 \mathrm{GHz}$ to $19.9 \mathrm{GHz}(108.5 \%$ bandwidth). Moreover, a $-3 \mathrm{~dB}$ RCSR is obtained nearly from $4.4 \mathrm{GHz}$ to $22 \mathrm{GHz}$ with a relative bandwidth of $133.3 \%$ for both TE and TM polarizations. For case 2, over $-6 \mathrm{~dB}$ RCSR bandwidth is $4.25 \sim 4.61 \mathrm{GHz}(8.13 \%$ bandwidth), 5.8 14.43 GHz (85.32\% bandwidth) and between 15.39 and $19.81 \mathrm{GHz}$ (25.11\% bandwidth), while over $-3 \mathrm{~dB}$ RCSR is achieved from $4.16 \mathrm{GHz}$ to $22 \mathrm{GHz}$ (136.39\% bandwidth). It is clearly demonstrated that case 2 exhibits better RCSR performance in the relatively low frequency band $(2 \sim 10 \mathrm{GHz})$ compared with case 1, whilst the frequencies of the three RCSR hollows shift slightly to lower frequency located at $4.4 \mathrm{GHz}, 6.9 \mathrm{GHz}$ and $10.1 \mathrm{GHz}$, respectively. On the contrary, in the relatively high frequency band $(10 \sim 16 \mathrm{GHz})$, case 1 presents a better performance in monostatic RCSR. The difference can boil down to the break of the periodicity by rotating the basic unit in case 2 .
In order to comprehensively illustrate the scattering performance of the finally proposed design (case 1), the monostatic RCS against frequency for TE- and TM-polarized normal incidence has been investigated, as shown in Fig. 5. It can be seen that the three peak RCSR points are obtained at $4.7 \mathrm{GHz}, 10.4 \mathrm{GHz}$ and $16.8 \mathrm{GHz}$ with a maximum RCSR value of $17.2 \mathrm{~dB}, 27.9 \mathrm{~dB}$ and $20.6 \mathrm{~dB}$, which is slightly different from the points of $180^{\circ}$ phase difference as depicted in Fig. 3. The discrepancy could be ascribed to the finite AMC unit arrangement. Moreover, the consistency between TE-polarization and TM-polarization can be attributed to the four-fold symmetric structure of each single sub-unit.

The simulated 2-D bistatic scattering pattern against the elevation angle has been explored under normal incidence at $4.7 \mathrm{GHz}, 10.4 \mathrm{GHz}$ and $16.8 \mathrm{GHz}$ separately for both TE and TM polarizations. For $\varphi=0^{\circ}$ cut plane, the simulated results of the proposed design are compared with an equal-sized metallic surface, as revealed in Fig. 6. It is evident that RCSR performance is achieved around boresight direction for all frequencies. The peak RCS is reduced by $6.8 \mathrm{~dB}, 11.9 \mathrm{~dB}, 15.6 \mathrm{~dB}$ at $4.7 \mathrm{GHz}, 10.4 \mathrm{GHz}$ and $16.8 \mathrm{GHz}$, respectively. Meanwhile, the reflected energy is divided into three main lobes at $\theta=61^{\circ}, 24^{\circ}, 14^{\circ}$ besides $0^{\circ}$ for $4.7 \mathrm{GHz}, 10.4 \mathrm{GHz}$ and $16.8 \mathrm{GHz}$. The maxima of the side lobes are reduced by $16.2 \mathrm{~dB}, 9 \mathrm{~dB}$ and $10.5 \mathrm{~dB}$ compared to the maximum of the corresponding metallic surface at these three frequencies. For $\varphi=45^{\circ}$ cut plane, the simulated bistatic RCS results with normal incidence has also been given. As plotted in Fig. 7, the RCS values of the proposed design decreased obviously around normal incidence direction for all three frequencies. The maximum RCS is reduced by $9.8 \mathrm{~dB}, 16.9 \mathrm{~dB}, 19.2 \mathrm{~dB}$ at 4.7 GHz, 10.4 GHz and $16.8 \mathrm{GHz}$, respectively. Since the incidence energy cannot be absorbed by the proposed AMC structure, the scattering field inevitably increases off the normal incidence direction as a result. Whereas, with the quadruple-triangular arrangement proposed in this paper, the scattering waves distribute more evenly over the entire space as a result of phase cancellation, which contributes a lot to the bistatic RCSR. As clearly observed along $\varphi=45^{\circ}$ plane, the incident waves are redirected into seven lobes and eleven lobes at $10.4 \mathrm{GHz}$ and $16.8 \mathrm{GHz}$, respectively. It is noteworthy that the redirected main lobes do not perfectly split in the same amplitude for both $\varphi=0^{\circ}$ and $45^{\circ}$. The reason is that the physical design geometry is not perfectly symmetric along these two planes together with the effect of edge diffraction.

To demonstrate diffusion effect of the proposed design more intuitively, the 3-D bistatic scattered patterns have also been exploited. The simulated 3-D bistatic scattered patterns at $4.7 \mathrm{GHz}, 10.4 \mathrm{GHz}$ and $16.8 \mathrm{GHz}$ are plotted in Fig. 8. We can see clearly that instead of a strong peak RCS, the scattered energy at $4.7 \mathrm{GHz}$ is redirected into five main lobes along $x o z$ and yoz planes, while that of $10.4 \mathrm{GHz}$ and $16.8 \mathrm{GHz}$ distribute more evenly with even more lobes over the entire space, resulting in a significant RCSR around the boresight direction as expected. 

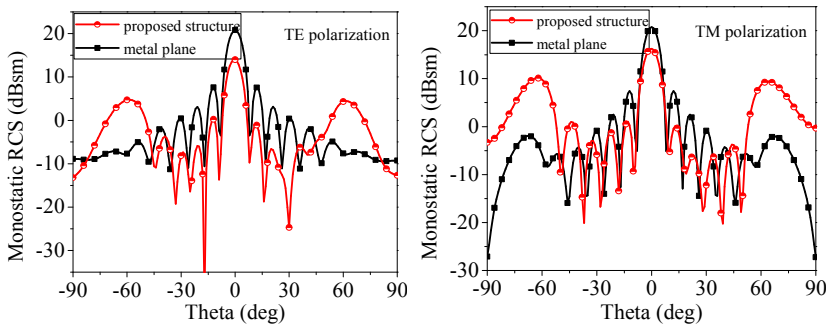

(a)
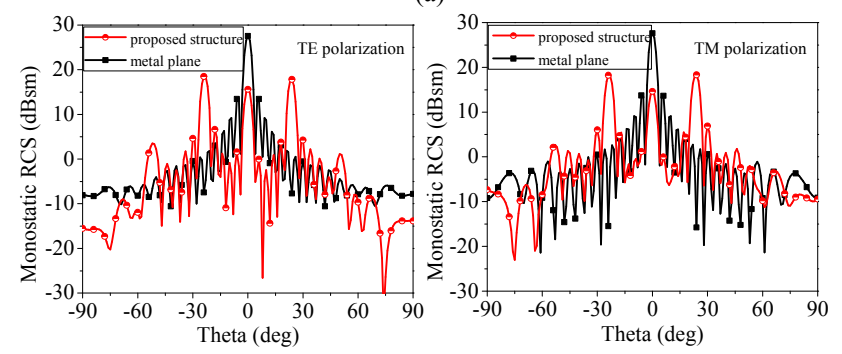

(b)
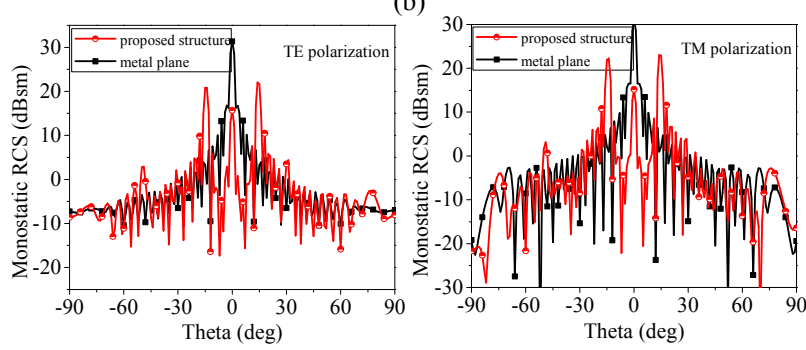

(c)

Fig. 6. Bistatic RCS versus angle for $\varphi=0^{\circ}$ cut plane under normal incidence at (a) $4.7 \mathrm{GHz}$; (b) $10.4 \mathrm{GHz}$ and (c) $16.8 \mathrm{GHz}$.
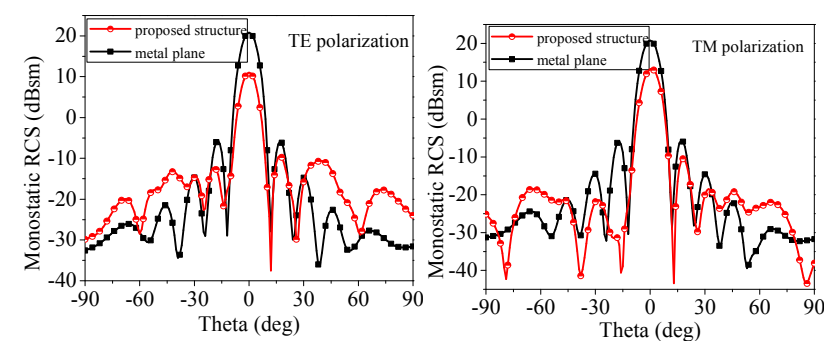

(a)
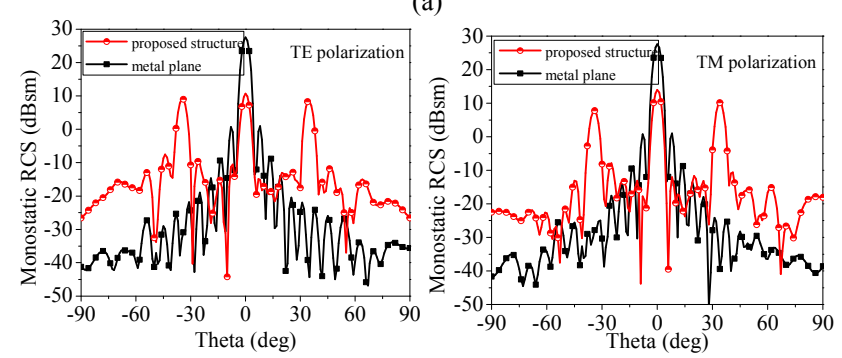

(b)
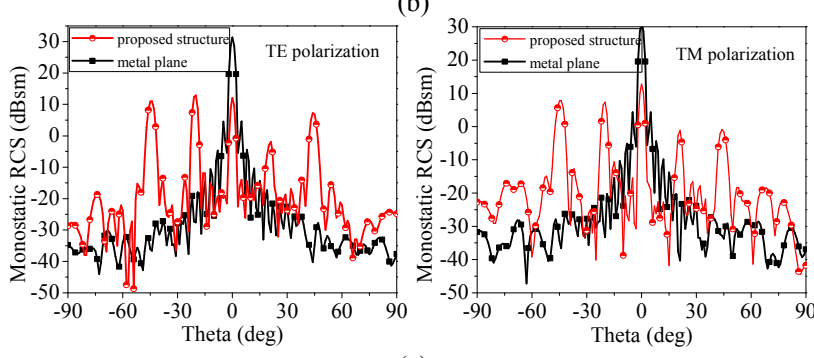

(c)

Fig. 7. Bistatic RCS versus angle for $\varphi=45^{\circ}$ cut plane under normal incidence at (a) 4.7; (b) 10.4 and (c) $16.8 \mathrm{GHz}$.

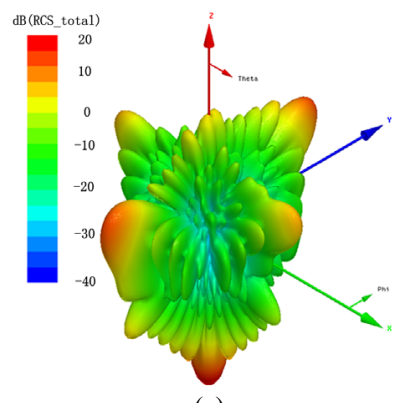

(a)

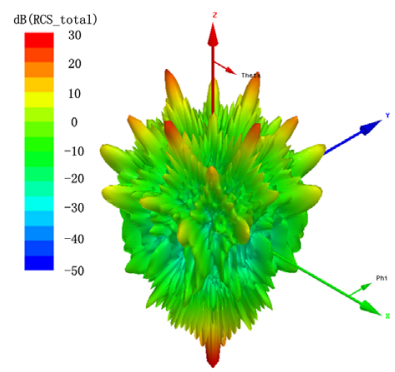

(b)

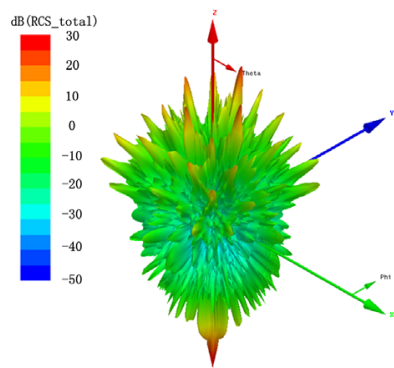

(c)

Fig. 8. Simulated 3D bistatic scattering patterns at: (a) $4.7 \mathrm{GHz}$; (b) $10.4 \mathrm{GHz}$ and (c) $16.8 \mathrm{GHz}$.

\section{Fabrication and Measurement}

In order to validate the simulated results and further verify the ultra-wideband RCSR characteristic, the prototype was fabricated and measured for normal incidence in a microwave anechoic chamber, as exhibited in Fig. 9. As clearly depicted, the structure was built on a F4B dielectric board through standard printed board technology with an overall dimension of $432 \times 432 \mathrm{~mm}^{2}$, and one period of the chessboard configuration consists of four triangular tiles with the three aforementioned AMC sub-units. The fabricated board was placed vertically on the center of a rotation table, while two identical linear-polarized pyramidal horn antennas working at $1-18 \mathrm{GHz}$ were placed adjacently as transmitter and receiver, respectively. Gate-reflect-line calibration in time-domain analysis kit of vector network analyzer (VNA) has been employed to further eliminate undesirable signals. The vector network analyzer Agilent N5230C was utilized to evaluate monostatic RCS value on transmission coefficients. Restricted by the experimental equipment, only the monostatic RCS value ranging from $2 \mathrm{GHz}$ to $18 \mathrm{GHz}$ has been measured. The measured monostatic RCS of the fabricated board was normalized by that of a same-sized metallic board. As shown in Fig. 10, 


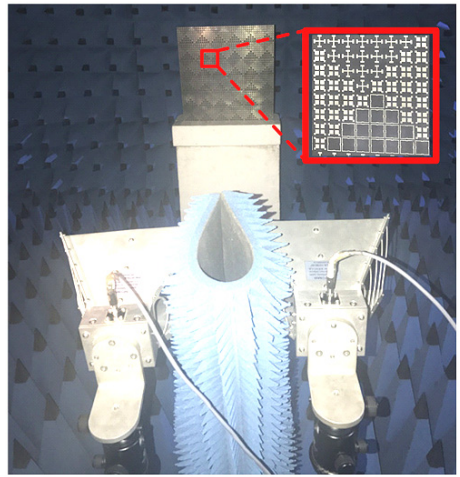

Fig. 9. Photograph of the fabricated prototype and the experimental setup.

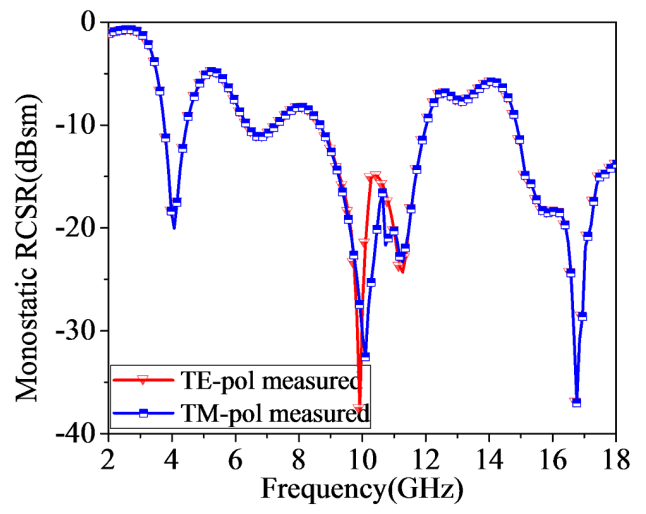

Fig. 10. Measured RCSR normalized by a same-sized metallic plate under normal incidence with TE and TM polarizations.

\begin{tabular}{|c|c|c|}
\hline Similar works & $\begin{array}{c}\text { Over }-6 \mathrm{~dB} \text { RCSR } \\
\text { frequency range }(\mathrm{GHz})\end{array}$ & Bandwidth (\%) \\
\hline$[4]$ & $14.1 \sim 23.1$ & 48.39 \\
\hline$[5]$ & $9.1 \sim 23.8$ & 89.36 \\
\hline$[6]$ & $8.9 \sim 17.8$ & 66.67 \\
\hline$[7]$ & $14 \sim 22.5$ & 46.58 \\
\hline This work & almost 3.62 18 & nearly 133.02 \\
\hline
\end{tabular}

Tab. 1. Experimental comparisons between this work and earlier similar works.

a -6dB RCSR is achieved from $3.62 \mathrm{GHz}$ to $18 \mathrm{GHz}$ except from $4.9 \mathrm{GHz}$ to $5.7 \mathrm{GHz}$ for both $\mathrm{TE}$ and $\mathrm{TM}$ polarizations. The peak RCSR value can reach up to $37.6 \mathrm{~dB}$. An excellent agreement between simulation and measurement verifies the ultra-wideband RCSR capability of the proposed design. The slight discrepancies result from the tolerance of fabrication and measurement. In order to highlight the advantages of our work, a measured results comparison between this work and earlier similar works has been made in Tab. 1, which clearly shows that the RCSR bandwidth is significantly enhanced by our design method.

\section{Conclusion}

A novel planar AMC structure has been designed, fabricated and measured for ultra-wideband RCSR. The structure consists of three different AMC tiles arranged in a quadruple triangle-type chessboard configuration. The three sub-unit cells are optimized to achieve destructive phase difference over an ultra-wideband nearly from $3.98 \mathrm{GHz}$ to $18.84 \mathrm{GHz}$. By this means, the scattered energy is redirected into several lobes to attenuate the peak scattered filed for the advantage of bistatic RCSR. And a measured -6dB RCSR is achieved nearly from $3.62 \mathrm{GHz}$ to $18 \mathrm{GHz}$ with a relative bandwidth of $133.02 \%$. Both simulated and measured results demonstrated that the proposed design performs the capability and potentiality for ultra-wideband RCSR. The proposed design provides a feasible approach to broaden the bandwidth of RCSR while achieving bistatic RCSR simultaneously. Moreover, the novel arrangement provides a novel way to address ultra-wideband RCSR of closely-spaced antenna array, which leads to the direction of our further work.

\section{Acknowledgments}

This work is partially supported by the National Natural Science Foundation of China (No. 61271100, No. 61471389, No. 61671464 and No. 61501494). All authors thank the reviewers for their valuable comments.

\section{References}

[1] PU, M. B., ZHAO, Z. Y., WANG, Y. Q., et al. Spatially and spectrally engineered spin-orbit interaction for achromatic virtual shaping. Scientific Reports, 2015, vol. 5, no. 9822, p. 1-6. DOI: 10.1038/srep09822

[2] WANG, K., ZHAO, J., CHENG, Q., et al. Broadband and broadangle low-scattering metasurface based on hybrid optimization algorithm. Scientific Reports, 2014, vol. 4, no. 5935, p. 1-5. DOI: 10.1038/srep05935

[3] GOMORY, F., SOLOVYOV, M., SOUC, J., et al. Experimental realization of a magnetic cloak. Science, 2012, vol. 335, p. 1466-1468. DOI: $10.1126 /$ science. 1218316

[4] DONG, D. S., YANG, J., CHENG, Q., et al. Terahertz broadband low-reflection metasurface by controlling phase distributions. Advanced Optical Materials, 2015, vol. 156, p. 1-6. DOI: 10.1002/adom.201500156

[5] LIU, X., GAO, J., XU, L. M., et al. A coding diffuse metasurface for RCS reduction. IEEE Antennas and Wireless Propagation Letters, 2017, vol. 16, p. 724-727. DOI: 10.1109/LAWP.2016.2601108

[6] ZHAO, Y., CAO, X. Y., GAO, J., et al. Broadband radar absorbing material based on orthogonal arrangement of CSRR etched artificial magnetic conductor. Microwave and Optical Technology Letters, 2014, vol. 56, no. 1, p. 158-161. DOI: $10.1002 /$ mop. 28033

[7] HUANG, C., PAN, W. B., MA, X. L., et al. Multi-spectral metasurface for different functional control of reflection waves. Scientific Reports, 2016, vol. 6, no. 23291, p. 1-7. DOI: $10.1038 /$ srep23291

[8] MIGHANI, M., DADASHZADEH G. Broadband RCS reduction using a novel double layer chessboard AMC surface. Electronics Letters, 2016, vol. 52, no. 14, p. 1253-1255. DOI: 10.1049/el.2016.1214 
[9] ZHAO, Y., CAO, X. Y., GAO, J., et al. A low-RCS and high-gain slot antenna using broadband metasurface. IEEE Antennas and Wireless Propagation Letters, 2016, vol. 15, p. 290-293. DOI: 10.1109/LAWP.2015.2442257

[10] PAQUAY, M., IRIARTE, J. C., EDERRA, I., et al. Thin AMC structure for radar cross-section reduction. IEEE Transactions on Antennas and Propagation, 2007, vol. 55, no. 12, p. 3630-3638. DOI: 10.1109/TAP.2007.910306

[11] SIMMS, S., FUSCO, V. Chessboard reflector for RCS reduction. Electronics Letters, 2008, vol. 44, no. 4, p. 316-317. DOI: 10.1049/el:20083368

[12] MONORCHIO, A., MANARA, G., LANUZZA, L. Synthesis of artificial magnetic conductors by using multilayered frequency selective surfaces. IEEE Antennas and Wireless Propagation Letters, 2002, vol. 1, no. 1, p. 196-199. DOI: 10.1109/LAWP.2002.807956

[13] DE COS, M. E., LAS-HERAS, F., FRANCO, M. Design of planar artificial magnetic conductor ground plane using frequencyselective surfaces for frequencies below $1 \mathrm{GHz}$. IEEE Antennas and Wireless Propagation Letters, 2009, vol. 8, p. 951-954. DOI: 10.1109/LAWP.2009.2029133

[14] CHEN, W. G., BALANIS, C. A., BIRTCHER, C. R. Dual wideband checkerboard surfaces for radar cross section reduction. IEEE Transactions on Antennas and Propagation, 2016, vol. 64, no. 9, p. 4133-4138. DOI: 10.1109/TAP.2016.2583505

[15] SAADAT, S., ADNAN, M., MOSALlAEI, H., et al. Composite metamaterial and metasurface integrated with non-foster active circuit elements: a bandwidth-enhancement investigation. IEEE Transactions on Antennas and Propagation, 2013, vol. 61, no. 3, p. 1210-1218. DOI: 10.1109/TAP.2012.2227654

[16] ZHANG, Y., MITTRA, R., WANG, B. Z., et al. AMCs for ultrathin and broadband RAM design. Electronics Letters, 2009, vol. 45, no. 10 , p. 484-485. DOI: 10.1049/el.2009.3161

[17] ZHENG, Y. J., GAO, J., CAO, X. Y., et al. Wideband RCS reduction of a microstrip antenna using artificial magnetic conductor structures. IEEE Antennas and Wireless Propagation Letters, 2015, vol. 14, p. 1582-1585. DOI: 10.1109/LAWP.2015.2413456

[18] CHEN, W. G., BALANIS, C. A., BIRTCHER, C. R. Checkerboard EBG surfaces for wideband radar cross section reduction. IEEE Transactions on Antennas and Propagation, 2015, vol. 63, no. 6, p. 2636-2645. DOI: 10.1109/TAP.2015.2414440

[19] IRIARTE, J. C., PEREDA, A. T., MARTINEZ DE FALCON, J. L., et al. Broadband radar cross-section reduction using AMC technology. IEEE Transactions on Antennas and Propagation, 2013, vol. 61, no. 12, p. 6136-6143. DOI: 10.1109/TAP.2013.2282915

[20] ESMAELI, S. H., SEDIGHY, S. H. Wideband radar cross-section reduction by AMC. Electronics Letters, 2016, vol. 52, no. 1, p. 70-71. DOI: 10.1049/el.2015.3515
[21] EDALATI, A., SARABANDI, K. Wideband, wide angle, polarization independent RCS reduction using nonabsorptive miniaturized-element frequency selective surfaces. IEEE Transactions on Antennas and Propagation, 2014, vol. 62, no. 2, p. 747-754. DOI: 10.1109/TAP.2013.2291236

[22] HONG, T., DONG, H., WANG, J., et al. A novel combinatorial triangle-type AMC structure for RCS reduction. Microwave and Optical Technology Letters, 2015, vol. 57, no. 12, p. 2728-2732. DOI: $10.1002 / \operatorname{mop} 29427$

[23] LI, M., XIAO, S., BAI, Y. Y., et al. An ultrathin and broadband radar absorber using resistive FSS. IEEE Antennas and Wireless Propagation Letters, 2012, vol. 11, p. 748-751. DOI: 10.1109/LAWP.2012.2206361

\section{About the Authors ...}

Tao SONG was born in Zaozhuang, Shandong province, P.R. China in 1990. He has received B.S. and M.S. from the Air Force Engineering University, Xi'an China, in 2013 and 2015, respectively. He is currently working toward Ph.D. degree at the Air and Missile Defense College, Air Force Engineering University. His research focuses on electromagnetic theory and application.

Lili CONG was born in Weihai, Shandong province, P.R. China in 1991. She received B.S. and M.S. from the Air Force Engineering University, Xi' an China, in 2013 and 2015. She is currently working toward Ph.D. degree at the Information and Navigation College, Air Force Engineering University. Her main interests include metamaterial design and wideband RCS reduction.

Chuangming TONG received the B. Sc and M.A. Sc degrees from the Air Force, the Missile Institute. He has more than 20-years-experience in teaching and research. $\mathrm{He}$ was engaged in postdoctoral research as a visiting scientist fellow with the Southeast University, Nanjing, China. $\mathrm{He}$ is currently a professor with the Dept. of Electronics and Communication Engineering, Air and Missile Defense College, Air Force Engineering University of CPLA. His research interests include microwave remote sensing, electromagnetic waves, polarimetric and interferometric applications of microwave data and numerical modeling, ground penetrating radar, through-wall imaging and stealth technology. Dr. Tong has received various fellowships and awards from national and international bodies so far. 